\title{
Long-Term Trends of P-Band Temporal Decorrelation Over a Tropical Dense Forest-Experimental Results for the BIOMASS Mission
}

\author{
Salma El Idrissi Essebtey ${ }^{\circledR}$, Ludovic Villard, Pierre Borderies, Thierry Koleck, Benoît Burban, and Thuy Le Toan
}

\begin{abstract}
Fostered by the upcoming BIOMASS mission, this article explores long-term trends of $\mathrm{P}$-band temporal decorrelation over a tropical forest due to a time series of 617 days acquired during the TropiScat-2 experiment. The interest in this unique time series is twofold. First, it provides consistent statistics to monitor the yearly evolution of temporal coherences according to specific time scales of the BIOMASS tomographic and interferometric phases. Second, it provides key insights to explore new processing approaches with the combination of data from different orbit directions (ascending/descending) and different mission cycles separated by about seven months according to the current acquisition plan. For the first time, this study shows that 18-day coherences (corresponding to the time interval between the first and last acquisitions of the BIOMASS tomographic processing) can vary significantly according to rainy and dry seasons (medians from 0.3 to 0.9 ). The extension to time intervals of up to 90 days within both seasons and over two consecutive years puts forward the key role of the typical sporadic rainfalls occurring during dry periods in tropical rainforests, with a stronger impact on temporal coherence evolution compared to the more reproducible rainy seasons. Furthermore, outstanding values significantly above zero have been obtained for the 7and 14-month coherences (medians of 0.35 and 0.2 , respectively), opening the way to new methods of change detection. Overall, this study highlights the role of P-band temporal decorrelation not only as a disturbance factor for coherent applications but also as a relevant indicator of forest changes.
\end{abstract}

Index Terms-BIOMASS mission, long-term temporal decorrelation, P-band, P-band scatterometer, repeat-pass polarimetric and interferometric synthetic-aperture radar (PolInSAR) and synthetic-aperture radar tomography (TomoSAR), tower-scat experiment, tropical dense forest.

Manuscript received November 10, 2020; revised March 20, 2021; accepted April 25, 2021. This work has been made possible with the support of the CNES research program referred to as TOSCA. The work of Salma El Idrissi Essebtey was supported in part by CNES and in part by the Office National d'Études et Recherches Aérospatiales (ONERA). (Corresponding author: Salma El Idrissi Essebtey.)

Salma El Idrissi Essebtey is with the Centre d'Études Spatiales de la BIOsphère (CESBIO), UMR CNES-CNRS-IRD-INRAE-UPS, 31401 Toulouse, France, and also with the Office National d'Études et Recherches Aérospatiales ONERA/DEMR, Université de Toulouse, 31055 Toulouse, France (e-mail: salma.el-idrissi-essebtey@univ-tlse3.fr).

Ludovic Villard, Thierry Koleck, and Thuy Le Toan are with the Centre d'Études Spatiales de la BIOsphère (CESBIO), 31401 Toulouse, France (e-mail: ludovic.villard@ cesbio.cnes.fr).

Pierre Borderies is with the Office National d'Études et Recherches Aérospatiales ONERA/DEMR, Université de Toulouse, 31055 Toulouse, France.

Benoît Burban is with the Ecologie des Forêts de Guyane (EcoFog) UMR AgroParisTech-CIRAD-INRA-CNRS-Université de Guyane-Universités des Antilles, 97379 Kourou, French Guiana.

\section{INTRODUCTION}

$\mathbf{T}$ HE last decade has witnessed an unprecedented number of experiments and studies based on P-band radar observations, resulting mostly from the preparation activities of the BIOMASS mission planned for launch in 2023. Beyond the technological challenges for the first P-band spaceborne SAR ever developed, the BIOMASS scientific objectives are also very demanding to reduce the current uncertainties in terrestrial carbon stock and flux estimates due to a better knowledge of the distribution of forest above-ground biomass (AGB) around the world [1]-[3]. The key mission products to address these objectives lie mainly on forest AGB and height maps at 4 ha resolution, characterized by uncertainties below $20 \%$ for tropical forests (see [2]). Being the longest wavelength possible for civilian spaceborne SAR, P-band SAR has demonstrated its unique capabilities for dense forest observations, whether related to its penetration capabilities through dense vegetation or its sensitivity to forest biomass [4], [5]. With regard to coherent techniques such as SAR interferometry or tomography, P-band is also recognized as the only frequency band possible from space allowing sufficiently high values of temporal coherences to perform relevant repeat-pass acquisitions beyond several days, over dense vegetation covers including tropical forests. The observations have been based on a tower-based scatterometer experiment known as TropiScat conducted from 2011 to 2014 over a test site of tropical forest in French Guiana [6]. The TropiScat experiment has provided key results regarding tropical forest monitoring at $\mathrm{P}$ and $\mathrm{L}$ bands by means of several time series of up to 45 days acquired with a 15-min time step [7], [8]. The analysis of these time series has confirmed the high level of P-band temporal coherences at one-day interval, with a mean value of about 0.8 based on several acquisition dates and times, whereas L-band mean coherence value has been evaluated at about 0.6. Moreover, it has highlighted that lower coherence values were found at 30 days both at $\mathrm{P}$ and $\mathrm{L}$ bands with mean values of about 0.6 and 0.4 , respectively [9]. These results confirm the high expectations on the use of P-band for repeat-pass configuration but also contribute to head for revisit intervals of three days between the interferometric passes, given the retained threshold of 0.8 in order to meet the forest height retrieval uncertainties better than $20 \%$ for dense forests [2], [10]. This interval of three days has been retained for both the tomographic phase (made of seven consecutive 
passes, during the first 14 months) and the subsequent dual baseline polarimetric and interferometric synthetic-aperture radar (PolInSAR) phase (made of a triplet of acquisitions, during the remaining four years of the mission). During both phases, P-band intensities will be available either from the tomographic cubes or from the more commonly used polarimetric and multitemporal stack of images. To compute these intensity images, an important processing step lies on speckle filtering [11], which efficiency depends on the absence of correlation between samples. Given the abovementioned high coherences expected between images separated by few days (as for a triplet), the independent (or weakly correlated) samples for the speckle filtering are constituted by polarization channels and spatial resolution cells [12], hence a resolution of about 4ha for the AGB product in order to meet the requirement of 20\% uncertainties for dense forests. The TropiScat time series have also emphasized significant variability in the evolution of coherences during the dry and rainy seasons, both in terms of the daily acquisition time and the considered time intervals [9], [13]. The experiment in French Guiana was later extended to another tropical forest in Africa between 2015 and 2017 , allowing the validation of the previously obtained results for another type of dense tropical forest [14], [15]. However, both experiments could not provide enough statistical samples to address the evolution of temporal decorrelation beyond one month. With the aim of extending these time series as well as completing the $\mathrm{P}$ and L-band observations with C-band acquisitions, a follow-on experiment referred to as TropiScat-2 has been set up since March 2018 over the same forest site as for TropiScat [16], [17]. A long time series of P-band measurements has been acquired quasi-continuously ever since. This nearly two-year time series enables to extend the previous studies about coherence evolution for longer time intervals reaching, for the first time, more than one year. Based on the exploitation of the aforementioned time series, this article is concerned with the investigation of long-term P-band coherences. These so-called long-term coherences can be considered as coherences computed for time intervals long enough to generate a potential risk of temporal decorrelation with regard to tomographic and interferometric applications. Considering the selected time constants for the tomographic phase of the BIOMASS mission, the threshold interval from which this assumption can be valid here is chosen at 18 days. On the one hand, the analysis of these long-term coherences enables to quantify coherence magnitudes relevant for the future BIOMASS mission. On the other hand, the analysis of multiple time scales and seasons can provide a better understanding of P-band coherence evolution.

This article is organized as follows. Section II recalls the experiment characteristics, presents the explored two-year database, and details the performed processing. Section III presents an overview of coherence evolution along with temporal baselines ranging from ten days to 14 months, based on the whole 617-day dataset. To better understand and dissociate the multiple sources of decorrelation, specific time intervals of high importance are then considered in the course of this article, starting in Section IV by the analysis of 18-day coherences. Section V is then dedicated to a seasonal analysis over two consecutive years through the study of coherences computed for time intervals spanning from one day and up to 90 days. Finally, we investigate more thoroughly the 7- and 12-month coherences in Section VI. Discussion and conclusions of the study are, respectively, given in Sections VII and VIII.

\section{Data Acquisition And Processing}

\section{A. Overview of the Experiment and Exploited Database}

In order to extend the P-band time series acquired during the TropiScat (2011-2015) [7], [8] and AfriScat (2015-2017) [14], [15] experiments as well as to investigate further aspects of temporal monitoring of a tropical forest, a new experiment named TropiScat- 2 has been conducted since March 2018 in Paracou, French Guiana. Identically as for the previous experiments [8], TropiScat-2 consists of a radar scatterometer formed by a network of 20 antennas constituting 17 receive-transmit antenna pairs installed on the top of a 55-m-high tower (about $20 \mathrm{~m}$ higher than the overall canopy). The system is fully polarimetric and designed with tomographic capabilities, not deployed throughout this article, providing measurements from all 17 antenna pairs every $15 \mathrm{~min}$ in the frequency bands $200-400 \mathrm{MHz}$ (P-band) and $800-1000 \mathrm{MHz}$ (close to L-Band). As part of TropiScat2 experiment, a new vector network analyzer (VNA, ref ENAE5063A detailed on http://www.keysight.com) covering a wide range from $5 \mathrm{kHz}$ to $6.5 \mathrm{GHz}$ has been incorporated in order to extend the bandwidth. This new VNA has been installed on the top of the tower unlike the previous experiments where the VNA was placed in the control room at the base of the tower. This choice was made in order to avoid the signal loss due to the use of very long RF cables along the tower. Another new feature of this experiment is the addition of several measurements in parallel with the P- and L-band measurements that also present in the previous campaigns, including the aforementioned C-band capabilities [16].

In this article, we focus on the analysis of P-band temporal coherences based on a 617-day time series acquired between April 1, 2018 and March 31, 2020. It is the longest time series acquired over the three experiments and is made up of a succession of different seasons over two years. It is worth mentioning that in French Guiana, seasons are classified as follows: a major rainy season running from April to July, followed by a major dry season spreading from August to October, then a minor rainy season from November to February, and finally a minor dry season lasting through March. These different seasons can change from one year to the other both in terms of the distribution and intensity of the rainfall amount within each of them, as well as in terms of their temporal boundaries, which can move forward or backward. The few measurement gaps identified in this nearly two-year time series are mainly due to on-site maintenance campaigns. For the present study, the only considered acquisition times of day are 6:00 A.M. and 6:00 P.M., which correspond to the local acquisition times of BIOMASS ascending and descending passes. More specifically, the data used to represent these two 
acquisition times are, respectively, considered between 5:30 A.M. and 6:30 A.M. and then between 5:30 P.M. and 6:30 P.M. in order to get more representative samples of what happens around these two particular times of the day. In addition to the P-band time series, we also rely in this article on rainfall and wind measurements, acquired every $30 \mathrm{~min}$ from the flux tower. These in situ data will be used for a cross analysis with the P-band temporal coherences to help in their interpretation.

\section{B. Postprocessing and Temporal Coherence Computation}

Considering that data acquisition from the VNA is in the frequency domain, the processing consists first in an inverse fast Fourier transform to convert the measurements into the time domain and consequently into the range domain. Each acquisition is thus transformed to a complex impulse response to which two corrections are applied. The first one consists in the use of the radar power budget equation to account for the antenna gains and the range propagation. The second correction concerns the compensation of the possible temporal variations impacting the coupling terms between antennas and the tower (including the antenna array). These two corrections are carried out as described in detail in [18], except for the compensation of the impulse response that is now performed for both the amplitude and the phase.

The resulting range impulse responses can then be used to compute the complex temporal coherence $\gamma$ and the intensity correlation $\Gamma$, respectively, according to the following formulas:

$$
\begin{aligned}
\gamma_{p q}\left(t_{1}, t_{2}\right) & =\frac{\sum_{k=1}^{K} \sum_{i m}^{i M} S_{t 1}^{k}(i) \cdot S_{t 2}^{k}(i)^{*}}{\sqrt{\sum_{k=1}^{K} \sum_{i m}^{i M}\left|S_{t 1}^{k}(i)\right|^{2} \sum_{k=1}^{K} \sum_{i m}^{i M}\left|S_{t 2}^{k}(i)\right|^{2}}} \\
\Gamma_{p q}\left(t_{1}, t_{2}\right) & =\frac{\sum_{k=1}^{K} \sum_{i m}^{i M} I_{t 1}^{k}(i) . I_{t 2}^{k}(i)}{\sqrt{\sum_{k=1}^{K} \sum_{i m}^{i M} I_{t 1}^{k}(i)^{2} \sum_{k=1}^{K} \sum_{i m}^{i M} I_{t 2}^{k}(i)^{2}}}
\end{aligned}
$$

where $I_{t}^{k}=\left|S_{t}^{k}(i)\right|^{2}-\left(\sum_{k=1}^{K} \sum_{i m}^{i M}\left|S_{t}^{k}(i)\right|^{2} / K \times(\mathrm{iM}\right.$-im + 1)), with " $i$ " being the range index between $i_{m}$ and $i_{M}$ that are the limits of the range of interest and $S_{t 1}$ and $S_{t 2}$ being the complex responses for acquisition times $t_{1}$ and $t_{2}$, respectively. The index " $k$ " serves to cover the 17 antenna pairs for each polarization $p q$ ( $p$ and $q$ are, respectively, the polarization of the receiving and transmitting antenna). It is worth noting that $i_{m}$ and $i_{M}$ are fixed throughout the study in such a way as to consider range distances comprised within horizontal distances of 45 and $60 \mathrm{~m}$ from the base of the tower as shown in Fig. 1, resulting in a limited range of incidence angle with respect to a virtual horizontal ground of $40.6^{\circ}-48.8^{\circ}$. In the follow-up, we will focus on studying the modulus of the complex temporal coherence, which we will refer to as coherence for simplification. In the course of this article, temporal coherences are mainly computed and statistically analyzed for a given temporal baseline $\Delta t$. In this case, all temporal coherences of which the two acquisition dates $t_{1}$ and $t_{2}$ are separated by a time interval of $\Delta t$ (i.e., $\left|t_{2}-t_{1}\right|=\Delta t$ ) are considered among the whole or a selected dataset. Also, when the reference time and the test time are mentioned, we are, respectively, referring to $t_{1}$ and $t_{2}$. Moreover, it should be noted that the cross-polarization coherence is

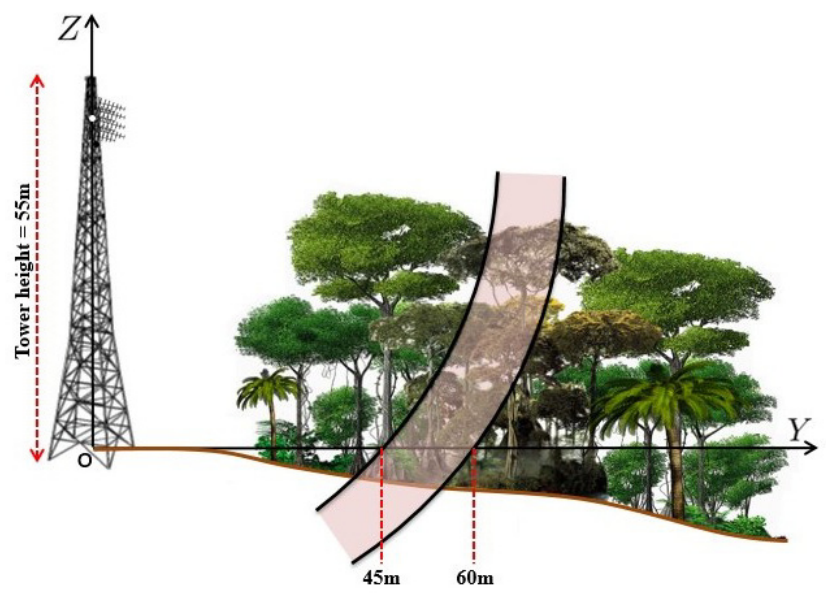

Fig. 1. Illustration of the observed forest site and the considered range area for the present study.

computed by combining both $\mathrm{HV}$ and $\mathrm{VH}$ as additional looks (since these channels convey the same information under the reciprocity principle) and will be referred to as HV coherence for simplification.

Regarding the resulting overall number of looks, it is worth to recall its importance in order to estimate the accuracy and precision of the provided coherences. A prior estimate based on the number of antenna pairs is not appropriate here given the spatial correlation between the iso-range cells needed for the tomographic capabilities of the experiment. Alternatively, a posterior estimate based on the squared average to variance ratio (common formula defining the equivalent number of looks (ENL) [11], [12]) is difficult to apply here due to the limited extent of the observed scene. Indeed, this formula requires a large and homogeneous area to ensure its convergence [19], [20]). Therefore, we propose to estimate the number of looks brought by the equivalent number of independent antenna pairs ( $\left.E N L_{\text {antenna-pairs }}\right)$ evaluated through the ratio between the overall ENL (resulting from the summation over all antenna pairs and spatial cells) and the mean value of ENL $_{\text {single-pair }}$ obtained from a single pair. The number of looks is then deduced by multiplying ENL antenna-pairs and the number of range cells within the considered interval $\left[y_{i m}=\right.$ $\left.45 \mathrm{~m}, y_{i M}=60 \mathrm{~m}\right]$ as resolved by the bandwidth, which is equal in our case to 14 range cells. Without anticipating the coherences analysis, we can stress according to [21] that the obtained median number of looks of about 50 can ensure a negligible bias for coherences above 0.2 and a bias below 0.1 for coherences below 0.2. It is also important to mention that a similar number of looks have been obtained for both the 6:00 A.M. and 6:00 P.M. measurements.

Before proceeding with the analysis of the forest temporal coherence, it is important to ensure that the system response remains stable throughout the entire considered period. Indeed, assuming the tower structure as a stable artificial target, its contribution can be isolated by the range resolution capabilities of the acquisitions and further can be used as a reference. The resulting temporal decorrelation is then a majoring value of the system one and can be used to assess its stability over time. When computed, the coherence values vary 

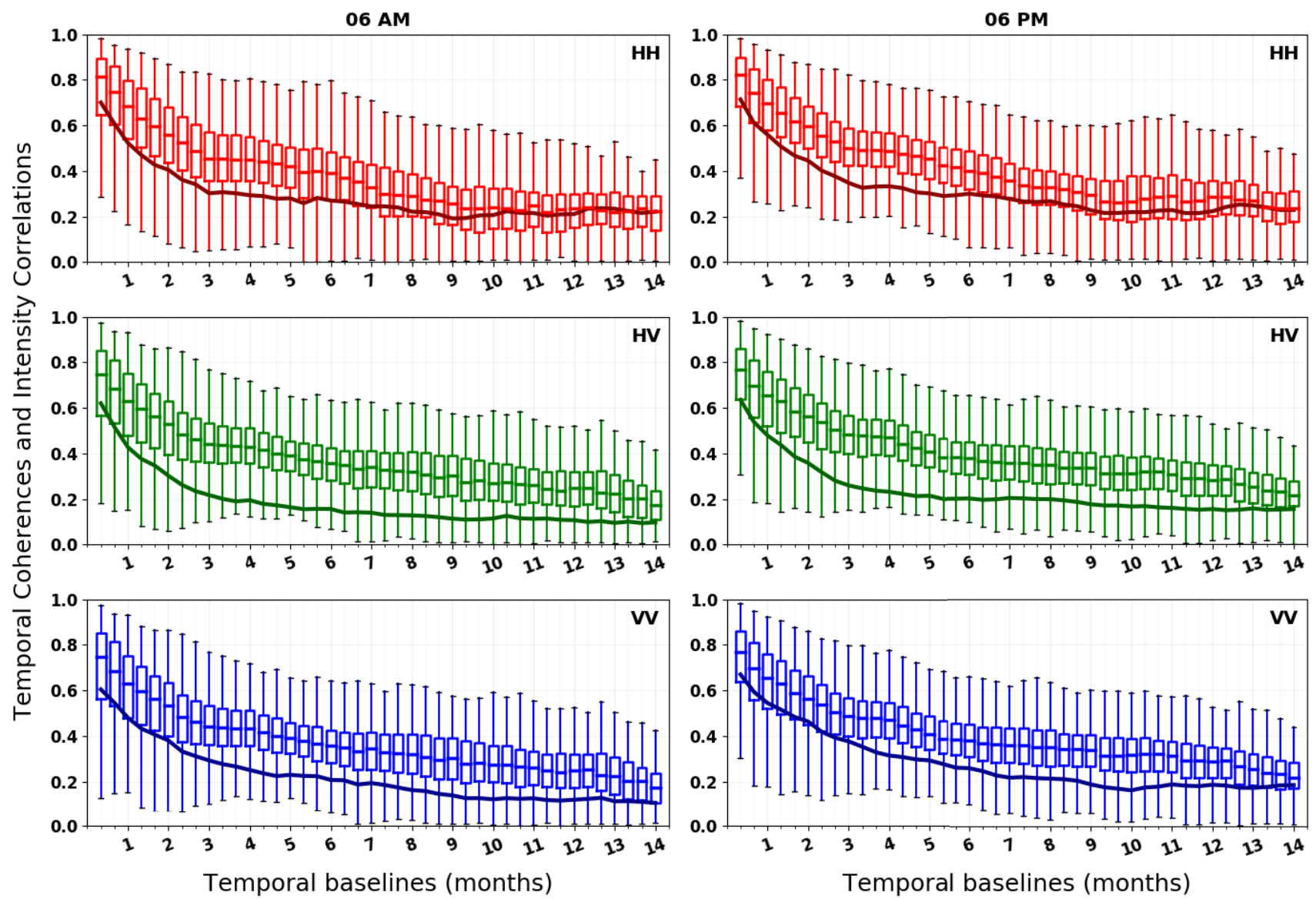

Fig. 2. Evolution of the statistical distribution of coherences (represented by whisker boxes) and medians of intensity correlations (continuous line curves) as a function of temporal baselines ranging from ten days to 14 months with a ten-day time step for (Left) 06:00 A.M. and (Right) 06:00 P.M. acquisitions and for the three polarizations $\mathrm{HH}, \mathrm{HV}$, and $\mathrm{VV}$ in each line.

around 0.9 for 14-month intervals, which is far higher than the forest coherence. Coherence values computed over the forest and presented throughout this study will not consider this system coherence and are therefore slightly underestimated. This choice was made to avoid the risk of overestimating the investigated coherence values.

\section{OVERVIEW OF TEMPORAL DECORRELATION AND INTENSITY CORRELATION FOR TIME INTERVALS OF UP TO 14 MONTHS}

\section{A. Temporal Decorrelation}

This first result section is mainly intended to provide an overview of coherence evolution with respect to the widest possible range of time intervals reaching for the first time 14 months, which corresponds to about two global cycles of BIOMASS acquisition scenario due to the aforementioned 617-day time series. The statistical distribution of coherences is of utmost importance in this mission context, hence the use of whisker boxes showing the percentage of coherences above a given threshold (minimum, first quartile, median, third quartile, and maximum). In Fig. 2, whisker boxes aim to characterize the set of coherence values resulting from all possible combinations of dates matching a given time interval indicated along the $x$-axis and spanning from ten days to 14 months with a time step of ten days. Coherences for both acquisition times are shown in the two columns of Fig. 2, while the three polarizations are shown on each line. From the outset, it can be noted that the decreasing evolution of temporal coherences is quite similar for all acquisition times and polarizations. Indeed, when using a decay model of the type of $a_{3} \cdot \exp \left(-a_{2} \cdot t\right)-a_{1} \cdot t^{2}+a_{0}$, we obtain very comparable regression parameters for both acquisition times and all three polarizations, as shown in Table I. This common decorrelation pattern can be characterized by three phases. First, this pattern follows an exponential decay model reflecting a decreasing speed of decorrelation up to about three months. The following phase consists of a rather linear trend characterized with a slight slope up to about nine months. This pattern ends finally with a stabilization phase where decorrelation no longer seems to be sensitive to temporal baselines. Yet, longer time series would be needed to confirm this hypothesis as indicated by the low drop that occurs from the 13-month temporal baseline for the VV polarization. To further analyze this evolution trend, it is worth recalling that decorrelation is a complex phenomenon that depends on several factors and possible sources. However, excluding sudden changes such as the ones resulting from anthropogenic actions or tree falls, one can distinguish between short-term decorrelation and long-term decorrelation. The first one can be due to wind gusts, heavy rains, and sudden changes in vegetation water content and soil moisture, whereas the second 
TABLE I

Regression PARAmeters For the Power-Law Model Relating Coherences and The CHOSEn Exponential DeCay Model

\begin{tabular}{|c|c|c|c|}
\hline & & 6:00 A.M. & 6:00 P.M. \\
\hline & $a_{0}$ & $\begin{array}{c}3.42 \mathrm{e}-01 \\
( \pm 1.99 \mathrm{e}-02)\end{array}$ & $\begin{array}{c}3.68 \mathrm{e}-01 \\
( \pm 2.08 \mathrm{e}-02)\end{array}$ \\
\hline & $a_{1}$ & $\begin{array}{c}8.68 \mathrm{e}-07 \\
( \pm 1.66 \mathrm{e}-07)\end{array}$ & $\begin{array}{c}8.16 \mathrm{e}-07( \pm 1.65 \mathrm{e}- \\
07)\end{array}$ \\
\hline $\mathrm{HH}$ & $a_{2}$ & $\begin{array}{c}1.58 \mathrm{e}-02 \\
( \pm 1.66 \mathrm{e}-03)\end{array}$ & $\begin{array}{c}1.44 \mathrm{e}-02 \\
( \pm 1.56 \mathrm{e}-03)\end{array}$ \\
\hline & $a_{3}$ & $\begin{array}{c}5.87 \mathrm{e}-01 \\
( \pm 2.35 \mathrm{e}-02)\end{array}$ & $\begin{array}{c}5.54 \mathrm{e}-01 \\
( \pm 2.24 \mathrm{e}-02)\end{array}$ \\
\hline & Chi2 & $2.92 \mathrm{e}-02$ & $2.68 \mathrm{e}-02$ \\
\hline & $\mathrm{a}_{0}$ & $\begin{array}{c}3.63 \mathrm{e}-01 \\
( \pm 8.71 \mathrm{e}-03)\end{array}$ & $\begin{array}{c}3.84 \mathrm{e}-01 \\
( \pm 1.04 \mathrm{e}-02)\end{array}$ \\
\hline & $a_{1}$ & $\begin{array}{c}7.09 \mathrm{e}-07 \\
( \pm 8.26 \mathrm{e}-08)\end{array}$ & $\begin{array}{c}5.34 \mathrm{e}-07 \\
( \pm 9.47 \mathrm{e}-08)\end{array}$ \\
\hline HV & $a_{2}$ & $\begin{array}{c}2.18 \mathrm{e}-02 \\
( \pm 1.31 \mathrm{e}-03)\end{array}$ & $\begin{array}{c}1.95 \mathrm{e}-02 \\
( \pm 1.35 \mathrm{e}-03)\end{array}$ \\
\hline & $a_{3}$ & $\begin{array}{c}5.75 \mathrm{e}-01 \\
( \pm 1.49 \mathrm{e}-02)\end{array}$ & $\begin{array}{c}5.48 \mathrm{e}-01 \\
( \pm 1.57 \mathrm{e}-02)\end{array}$ \\
\hline & Chi2 & $1.85 \mathrm{e}-02$ & $1.97 \mathrm{e}-02$ \\
\hline & $\mathrm{a}_{0}$ & $\begin{array}{c}3.97 \mathrm{e}-01 \\
( \pm 8.33 \mathrm{e}-03)\end{array}$ & $\begin{array}{c}4.16 \mathrm{e}-01 \\
( \pm 1.01 \mathrm{e}-02)\end{array}$ \\
\hline & $a_{1}$ & $\begin{array}{c}1.23 \mathrm{e}-06 \\
( \pm 8.29 \mathrm{e}-08)\end{array}$ & $\begin{array}{c}1.09 \mathrm{e}-06( \pm 9.60 \mathrm{e}- \\
08)\end{array}$ \\
\hline VV & $a_{2}$ & $\begin{array}{c}2.56 \mathrm{e}-02 \\
( \pm 1.75 \mathrm{e}-03) \\
\end{array}$ & $\begin{array}{c}2.21 \mathrm{e}-02 \\
( \pm 1.74 \mathrm{e}-03) \\
\end{array}$ \\
\hline & $a_{3}$ & $\begin{array}{c}5.40 \mathrm{e}-01 \\
( \pm 1.69 \mathrm{e}-02)\end{array}$ & $\begin{array}{c}5.13 \mathrm{e}-01 \\
( \pm 1.75 \mathrm{e}-02)\end{array}$ \\
\hline & Chi2 & $2.03 \mathrm{e}-02$ & $2.17 \mathrm{e}-02$ \\
\hline
\end{tabular}

one is mainly related to forest growth and cumulative rainfall and soil moisture exhibited by seasonal changes. If we exclude short-term and quasi-random sources of decorrelation, even though they are the most frequent during rainy seasons, we can hypothesize that the obtained decorrelation pattern is mostly attributed to the duration and succession of dry and rainy seasons and the distribution of rainy episodes. Indeed, when both the reference and test times belong to the same season, the obtained coherences are high enough to be sensitive to the ten-day increment and hence the high rate of decorrelation in the first phase of evolution. Nevertheless, when the two dates belong to different seasons, the resulting coherences are lower and, therefore, less sensitive to the increase of the temporal baselines and hence the reduced decreasing slope in the two later phases. This hypothesis will be verified in Sections V and VI with the analysis of subperiods characterized by specific conditions, allowing to isolate decorrelation sources and to evaluate their effects.

\section{B. Intensity Correlation}

Before pursuing the investigation of the coherence evolution, the current study is also an opportunity to address a very wide dynamic range of intensity correlation, defined by the real correlation coefficient between intensity values at two dates [see (2)]. Intensity correlations are of capital importance for the multitemporal filtering of backscattering coefficients. Indeed, the latter enables to minimize the use
TABLE II

Regression Parameters For the Power-Law MOdel Relating COHERENCES AND INTENSITY CORRELATIONS

\begin{tabular}{|c|c|c|c|c|}
\hline \multicolumn{2}{|c|}{} & $\mathrm{HH}$ & $\mathrm{HV}$ & $\mathrm{VV}$ \\
\hline \multirow{3}{*}{$6: 00$ A.M. } & $\alpha$ & $1.387 \pm$ & $1.752 \pm$ & $1.551 \pm$ \\
& & $0.486 \%$ & $0.532 \%$ & $0.501 \%$ \\
\cline { 2 - 5 } & Chi-square & 10.248 & 6.662 & 8.438 \\
\hline \multirow{3}{*}{$6: 00$ P.M. } & $\alpha$ & $1.368 \pm$ & $1.645 \pm$ & $1.395 \pm$ \\
& & $0.394 \%$ & $0.471 \%$ & $0.442 \%$ \\
\cline { 2 - 5 } & Chi-square & 7.216 & 6.643 & 8.849 \\
\hline
\end{tabular}

of spatial filtering, commonly based on box-car windowing, by using temporal looks and preserves thereby the spatial resolution. As stated in many previous works [22]-[24], the efficiency of multitemporal filtering is strongly dependent on the covariance matrix gathering the intensity correlations from all combinations of temporal pairs included in the ingested time series. As shown in [12], an optimal formulation dedicated to partially correlated images can be derived. Based on this formulation and the present experimental values, we can confirm that the ENL gain with a BIOMASS triplet (three passes at $t, t+3$, and $t+6$ days) is not really relevant since it provides a multiplicative factor of 1.17 (instead of 3 in the case of independent acquisitions). More interesting is the combination of two triplets separated by a global cycle of seven months, which provides a multiplicative gain of 1.63 ( 2 if independent) with an observed value around 0.2 for the seven-month intensity correlation median. Between these two cases (six days and seven months), the ENL gain can be very variable in view of the observed exponential decay, providing relevant figures to anticipate the combination of ascending and descending passes whose separation time is very dependent on latitude and longitude and can vary from few days to three months, according to the current BIOMASS acquisition plan. To close this section, it is worth highlighting the link between both temporal coherence and intensity correlation since it is possible to obtain a very relevant quality of fit with a simple power low model $(\alpha y=x)$ to relate their respective medians. Indeed, as shown in Table II, gathering the regression parameters with uncertainties and chi-square coefficients for all polarizations and both acquisition times, we can note rather accurate estimation with uncertainties below $0.4 \%$. To better understand the polarization dependence as well as the behavior of their mutual dispersion, further theoretical analysis would be very interesting but beyond the scope of the present study.

\section{18-DAy COHEREnCES AnAlysis}

As mentioned above, specific time scales can be chosen to dissociate the multiple causes of temporal decorrelation, starting with a temporal baseline of 18 days. The latter can be considered in this study as a transition between short- and long-term temporal baselines, in addition to being a key time scale of the tomographic phase of the BIOMASS mission. Indeed, the BIOMASS tomographic acquisition mode has been planned with seven three-day passes, resulting in a separation time of 18 days between the first and the last pass forming each tomographic acquisition. Although this technique is very 

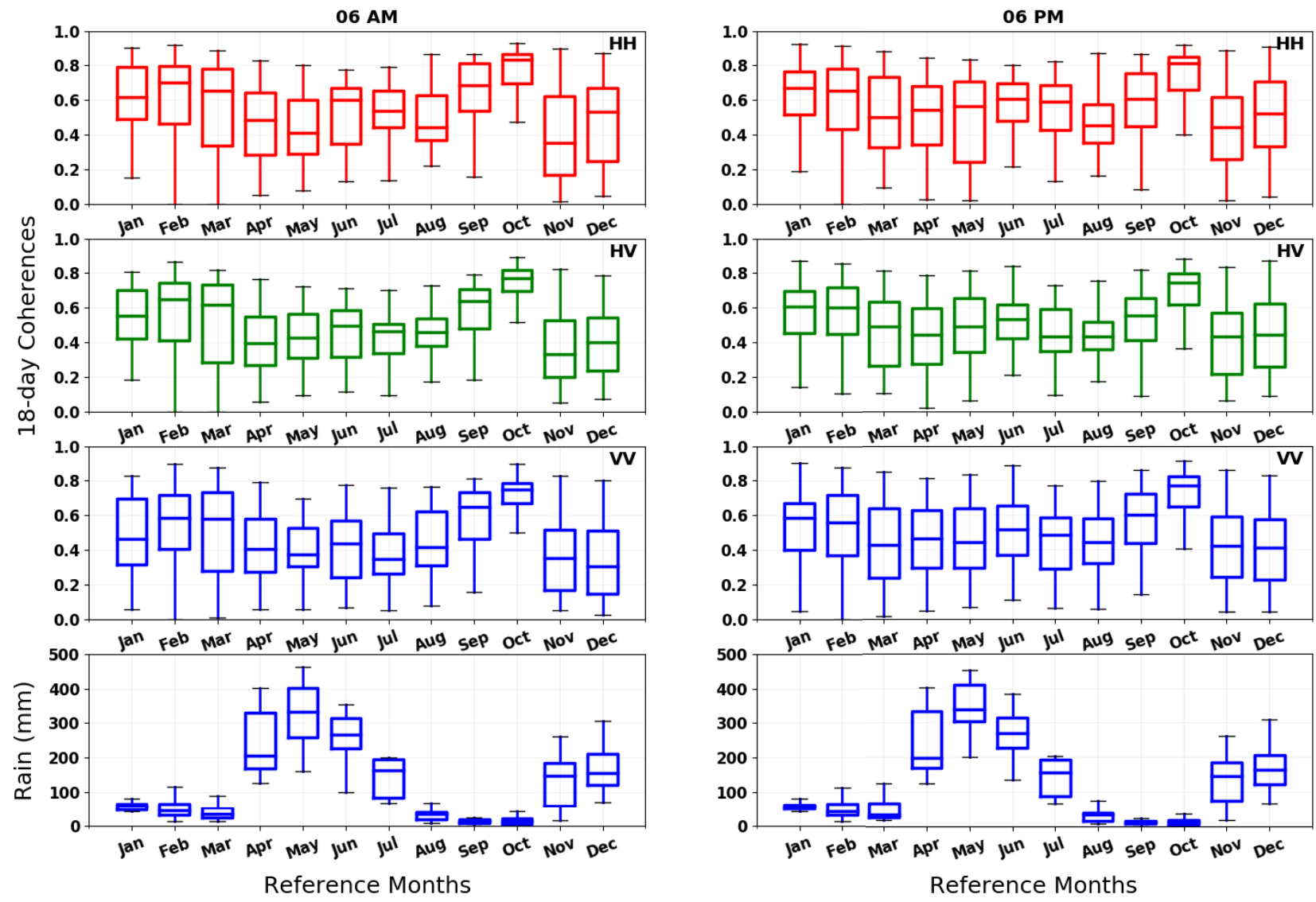

Fig. 3. Evolution of whisker boxes representing the statistical distribution of 18-day coherences (three top graphs for HH, HV, and VV polarizations) and 18-day cumulative rainfall (bottom graph) as a function of the reference month over the year, for (Left) 06:00 A.M. and (Right) 06:00 P.M.

innovative, it is important to note that throughout the entire mission lifetime, only a single pair of ascending/descending tomographic products will be available at a given latitude and longitude. It will therefore be difficult to mitigate the disruptive effects of local environmental conditions, in particular the effects of a varying temporal decorrelation. Consequently, the yearly distribution of 18-day coherences is of particular interest to help address this issue. Fig. 3 shows the annual distribution of whisker boxes constituted by all acquisition pairs separated by a time interval of 18 days among the 617-day time series, in which reference times fall into the month indicated along the $x$-axis. Overall, we can notice a significant variability during the year, whether for the median values or the dispersion. The medians vary mostly between 0.4 and 0.8 with a minimum value of about 0.3 (on December at 6:00 A.M.), justifying thereby their recognition as a transition to long-term coherences. Based on the previously mentioned exponential decreasing model in Section III, it is worth mentioning that an 18-day coherence of 0.6 , for instance, means that about half of the seven tomographic passes are likely to be impacted by temporal decorrelation, assuming a common threshold value of 0.8 . Besides, we can notice a very clear and common linear increase from August to October, for all polarizations and both acquisition times, coinciding with the major dry season. This pattern is then interrupted by a drop in November leading to a sort of a stable plateau of about two months for $\mathrm{VV}$ and HV polarizations and a more fluctuating one for $\mathrm{HH}$. This plateau is then followed by a slightly increasing trend up to February and March for, respectively, 6:00 P.M. and 6:00 A.M., before forming a more or less pronounced hollow ending with a local peak in June. Considering the distribution of the 18-day cumulative rainfall shown at the bottom of Fig. 3 in the same way as for the 18-day coherences, the undulating pattern of the latter becomes clearer, although the major rainy season of April and May seems to have less impact on the evolution of coherences than the minor rainy season of November and December. This observation indicates an integrative process related to past events whereby rainy events occurring after a long dry period have a greater effect on the evolution of temporal decorrelation than those occurring after a minor rainy season followed by a minor dry one, even though they are more intense. Furthermore, the very similar patterns between 6:00 A.M. and 6:00 P.M. coherences are an important indication of the predominant effects of progressive changes in the forest evolution in comparison to quasi-random or sudden disturbances, considering the stronger wind values recorded at 6:00 P.M. (as shown in Fig. 4). Nonetheless, differences can be still noted around March and May and June, which makes the phase opposition with the monthly rainfall distribution more obvious at 6:00 A.M., although no clear relation has been found with possible unbalanced rainfall between 6:00 A.M. 

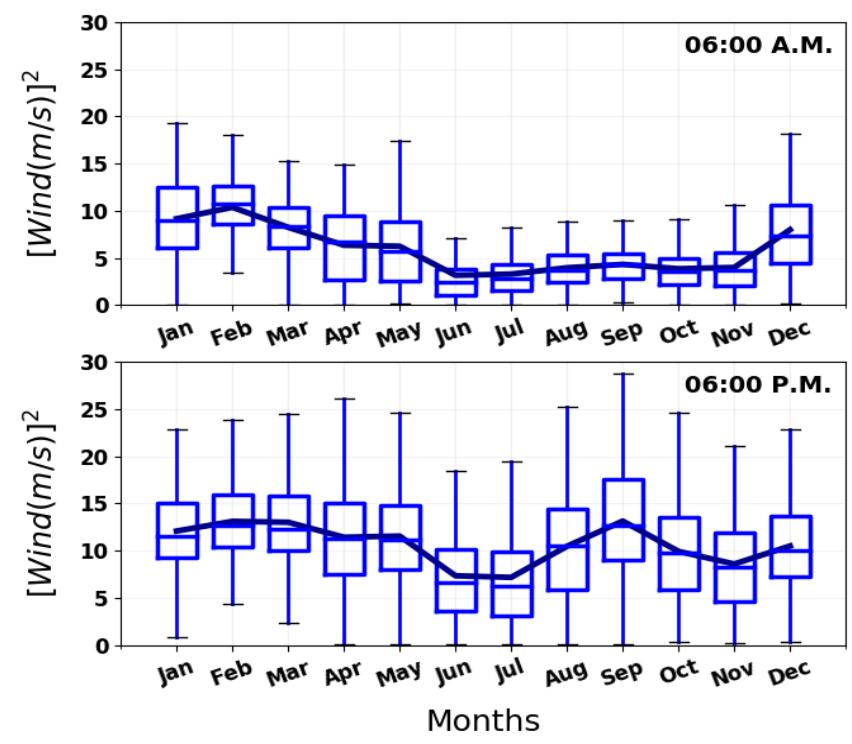

Fig. 4. Yearly evolution of whisker boxes representing the statistical distribution of 30-min mean wind velocity squared measurements as a function of the month for (Top) 6:00 A.M. and (Bottom) 6:00 P.M.

TABLE III

CORRELATION COEFFicients BETWEen 18-MONTH COHERENCES Calculated at 6:00 A.M. AND 6:00 P.M. AND Classified By THE REFERENCE MONTH. VALUES IN BOLD CORRESPOND TO THE SIGNIFICANT ONES ACCORDING TO A SIGNIFICANCE TEST WiTH A 5\% RISK

\begin{tabular}{|c|c|c|c|}
\hline & $\mathrm{HH}$ & $\mathrm{HV}$ & $\mathrm{VV}$ \\
\hline Jan & -0.104 & -0.0979 & -0.0647 \\
\hline Feb & $\mathbf{0 . 1 3 4}$ & $\mathbf{0 . 1 8 1}$ & $\mathbf{0 . 1 1 8}$ \\
\hline Mar & $\mathbf{0 . 2 7 5}$ & $\mathbf{0 . 1 8 9}$ & $\mathbf{0 . 3 3 0}$ \\
\hline Apr & 0.0571 & -0.0168 & 0.0491 \\
\hline May & -0.0593 & $\mathbf{0 . 2 7 2}$ & -0.0283 \\
\hline Jun & $\mathbf{0 . 2 0 7}$ & $\mathbf{0 . 2 2 3}$ & -0.0159 \\
\hline Jul & 0.155 & 0.0190 & -0.0380 \\
\hline Aug & $\mathbf{0 . 5 9 9}$ & $\mathbf{0 . 4 7 4}$ & $\mathbf{0 . 7 3 1}$ \\
\hline Sep & 0.0784 & 0.0843 & $\mathbf{0 . 1 5 1}$ \\
\hline Oct & $\mathbf{0 . 2 4 1}$ & $\mathbf{0 . 3 6 3}$ & $\mathbf{0 . 3 2 3}$ \\
\hline Nov & $\mathbf{0 . 4 7 1}$ & $\mathbf{0 . 4 4 8}$ & $\mathbf{0 . 4 7 3}$ \\
\hline Dec & $\mathbf{0 . 2 0 5}$ & $\mathbf{0 . 2 1 7}$ & $\mathbf{0 . 1 2 8}$ \\
\hline
\end{tabular}

and 6:00 P.M. Beyond the pattern analysis between 18-day coherences at 6:00 A.M. and 6:00 P.M, it is also very interesting to note the variability (from January to December) of the correlation values between both, as synthesized in Table III. To interpret these values, we can stress that the significant values reflect the occurrence of days for which the upper (respectively, lower) values of coherences at 6:00 A.M. correspond to upper (respectively, lower) values at 6:00 P.M. Although we cannot fully explain these correlation values, the intra-annual sensitivity demonstrates that these correlations can be a relevant indicator of seasonality effects.

\section{Seasonality EFFects on Temporal DeCORRELATION OVER TWO CONSECUTIVE YeARS FOR INTERVALS OF UP TO 90 DAYS}

In view of the importance of seasonality effects highlighted above as well as in previous works with temporal baselines
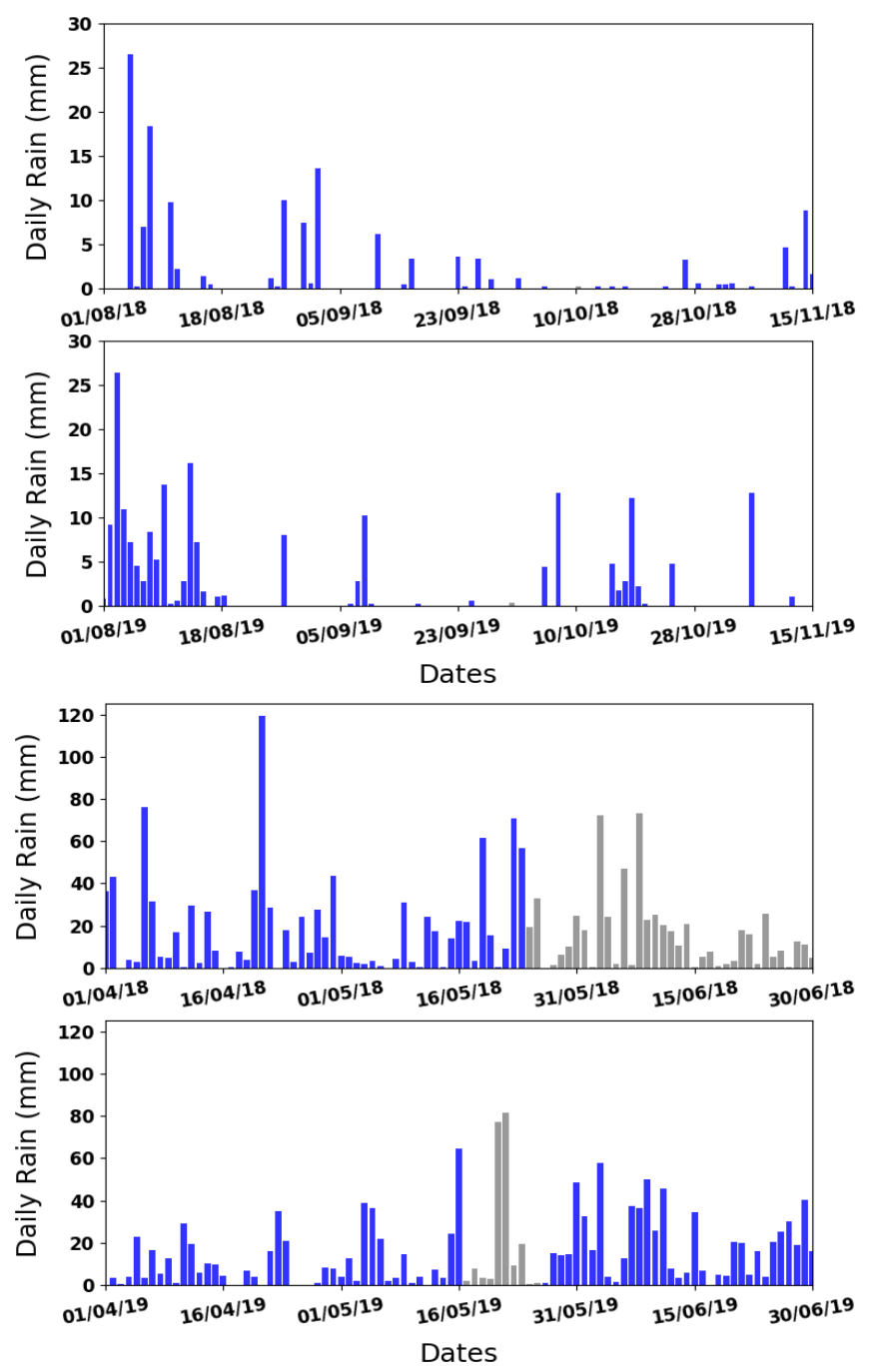

Fig. 5. Daily cumulative rainfall distribution over the four selected periods: (from Top to Bottom) 2018 dry season, 2019 dry season, 2018 rainy season, and 2019 rainy season. The gray bars correspond to days with no radar measurements, either because of an on-site mission or a technical problem.

of 30 days [9], the purpose of this section is to capitalize on the present database in order to investigate the longest possible temporal baselines within the same season, particularly the major dry and rainy seasons of 2018 and 2019.

\section{A. Description of the Selected Periods}

To derive the longest possible intervals within the major dry and rainy consecutive seasons, we first consider two 101-day time series over the major dry period of 2018 and 2019, namely the one extending from August 1 to November 15. These two dry periods are characterized, respectively, by a cumulative rainfall of about 140.2 and $202.6 \mathrm{~mm}$ and an almost equal number of dry days (i.e., days with a zero daily cumulative rainfall). However, the daily rainfall distribution is different from one dry period to another, as shown with the two top graphs of Fig. 5. The two rainy periods involved in this study cover from April 1, 2018 to May 24, 2018 and from April 1, 2019 to June 30, 2019. The interruption of the 2018 rainy period measurements was forced by a system outage that was overcome during an on-site mission the following 

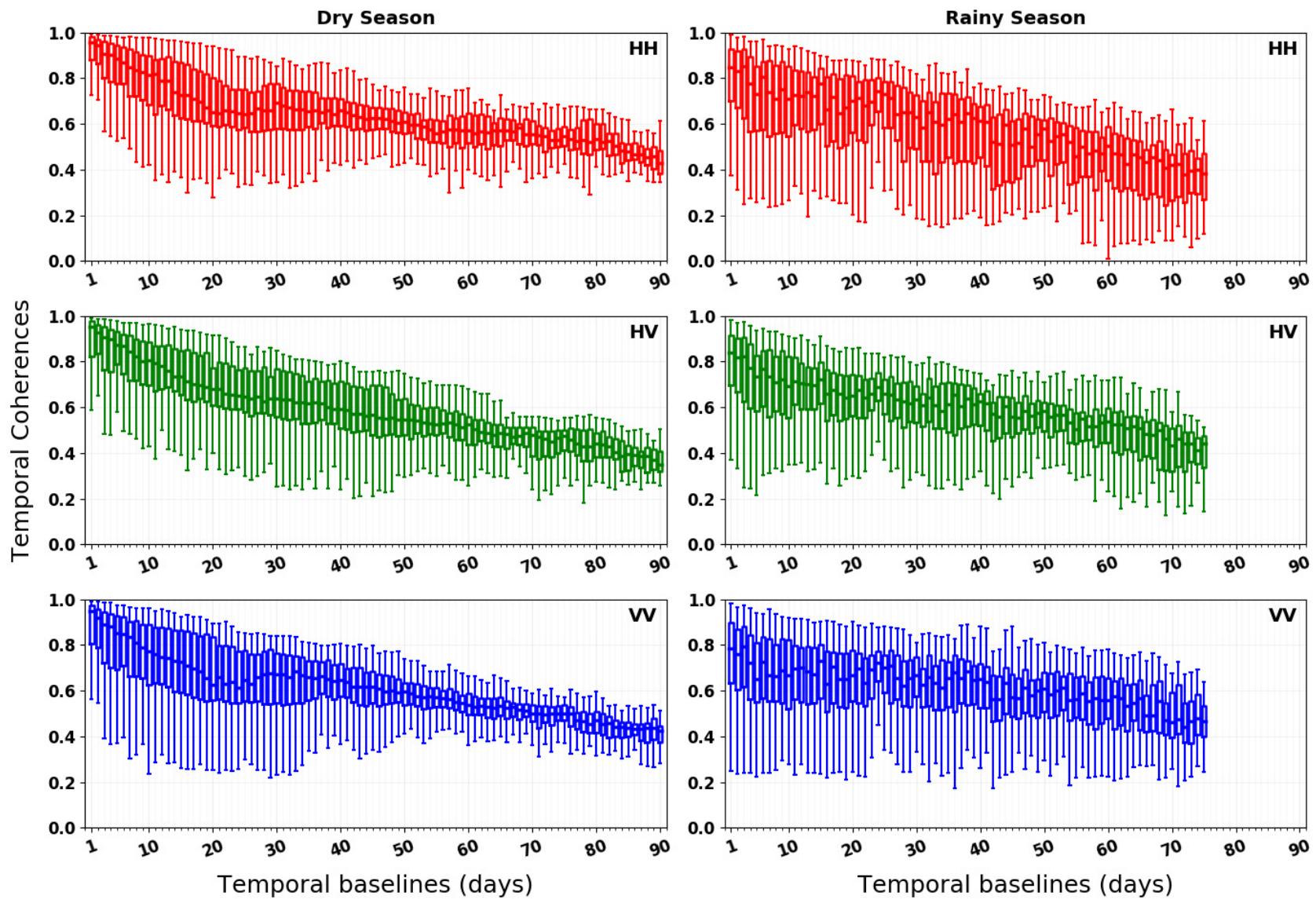

Fig. 6. Evolution of whisker boxes representing the statistical distribution of coherences as a function of temporal baselines ranging from one day to up to 90 days with a one-day time step for 6:00 A.M. acquisition time, over (Left) 2019 dry season and (Right) rainy one, and for the three polarizations HH, HV, and VV in each line.

summer. These two major rainy periods are characterized, respectively, by a cumulative rainfall of about 1573.6 and $1389.8 \mathrm{~mm}$ with a highly fluctuating daily distribution for both periods, as shown in the two bottom graphs of Fig. 5 . Regarding wind characterization, the distribution of 30-min averaged wind measurements is practically the same for both years over each season, with higher measured values during the rainy seasons than during the dry ones.

\section{B. Results and Analysis}

In this section, we present the evolution of the coherence distribution by means of whisker boxes as performed in Section III, considering this time coherences computed over the four selected seasons for temporal baselines ranging from one day to up to 90 days with a one-day time step. The comparison between distributions extracted from the dry and rainy periods of 2019 can be made through the left and right of Fig. 6, displaying one polarization per line. More specifically, the evolution of median values over the two consecutive years (2018 and 2019) is then overlapped in Fig. 7 , showing the three polarizations and both periods, respectively, per line and column. Based on both figures, we can first retrieve the typical difference between dry and rainy periods due to the steeper drop occurring after one day in the rainy cases, as previously highlighted in [9] and [13]. Besides, the reduced dispersion and the absence of the one-day drop during the dry periods result in a stronger and a more linear decreasing trend up to about 20 days, yet with significantly different slope magnitudes between both years. The occurrence or not of slope breaks, characterizing also both dry periods, will be further elaborated later in this section. Given these typical features from the early evolution of coherences, median values over dry periods remain mostly higher than those over rainy ones. However, these differences become less and less pronounced as the temporal baselines increase until about 45-day intervals where almost all medians fall within the range $0.5-0.7$. Considering the dry and rainy periods separately, it is interesting to note that the observed discrepancies between medians during the rainy periods can be more related to the time of acquisition (6:00 A.M. or 6:00 P.M.) than to the selected year. Conversely, dry periods are clearly more sensitive to the selected year up to about 60 days, from which the effects of acquisition times become significant as well. This noticeable long-term feature will be further analyzed in Section VI.

To better understand the afore-mentioned slope breaks occurring over the dry seasons (around 25 and 40 days for 2019 and around 33 and 65 days for 2018), coherences can be represented directly as a function of time, instead of variable time intervals. To do so, we start by selecting three reference times spread over the 2019 dry season (August 22, September 15, and November 13 at 6:00 A.M.), and then, we compute the evolution of coherences over the whole season 

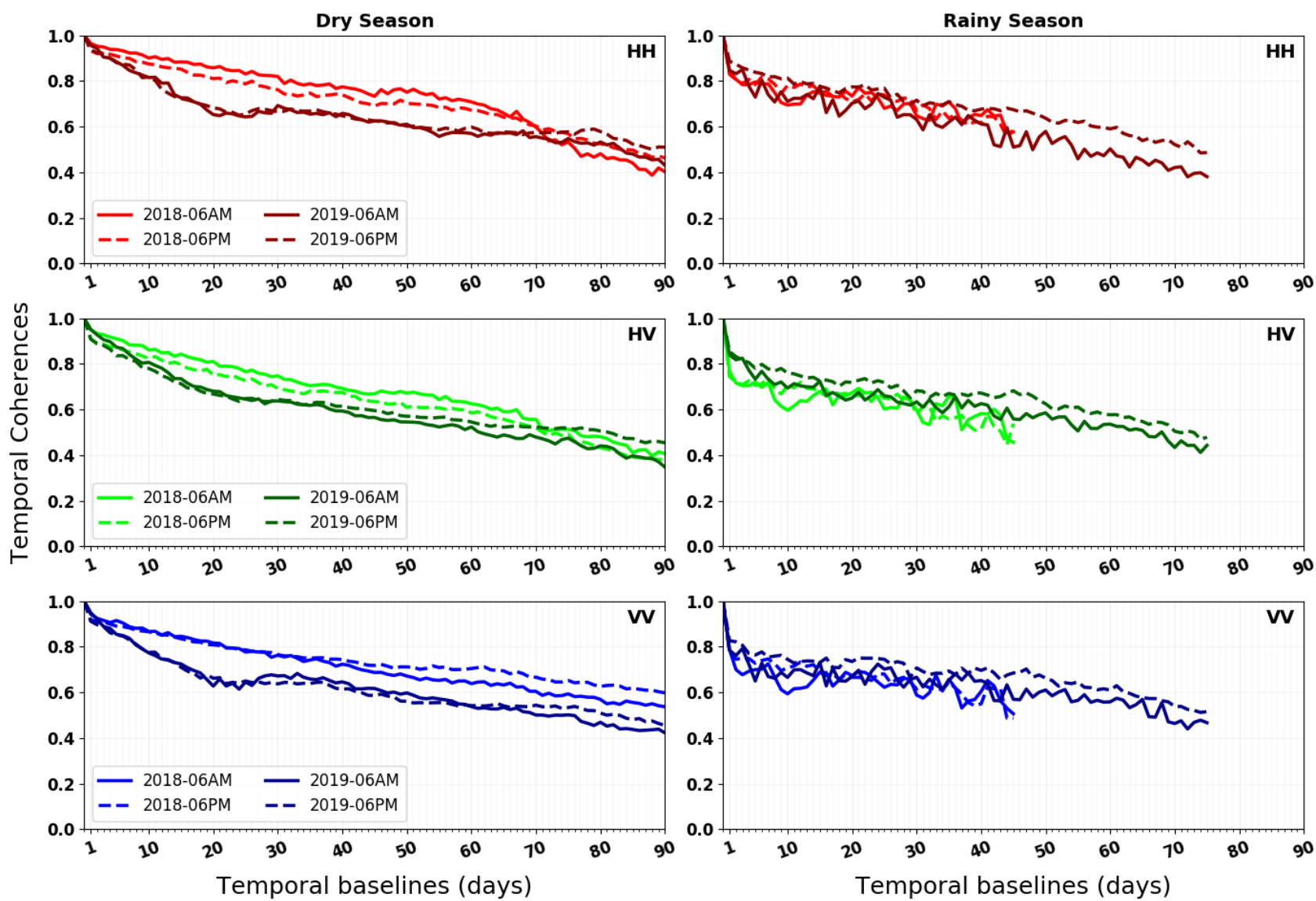

Fig. 7. Evolution of coherence medians as a function of temporal baselines ranging from one day to up to 90 days with a one-day time step, over 2018 (light colors) and 2019 (dark colors) (Left) dry and (Right) rainy seasons for both 6:00 A.M. (continuous line) and 6:00 P.M. (dashed line) and the three polarizations $\mathrm{HH}, \mathrm{HV}$, and $\mathrm{VV}$ in each line.

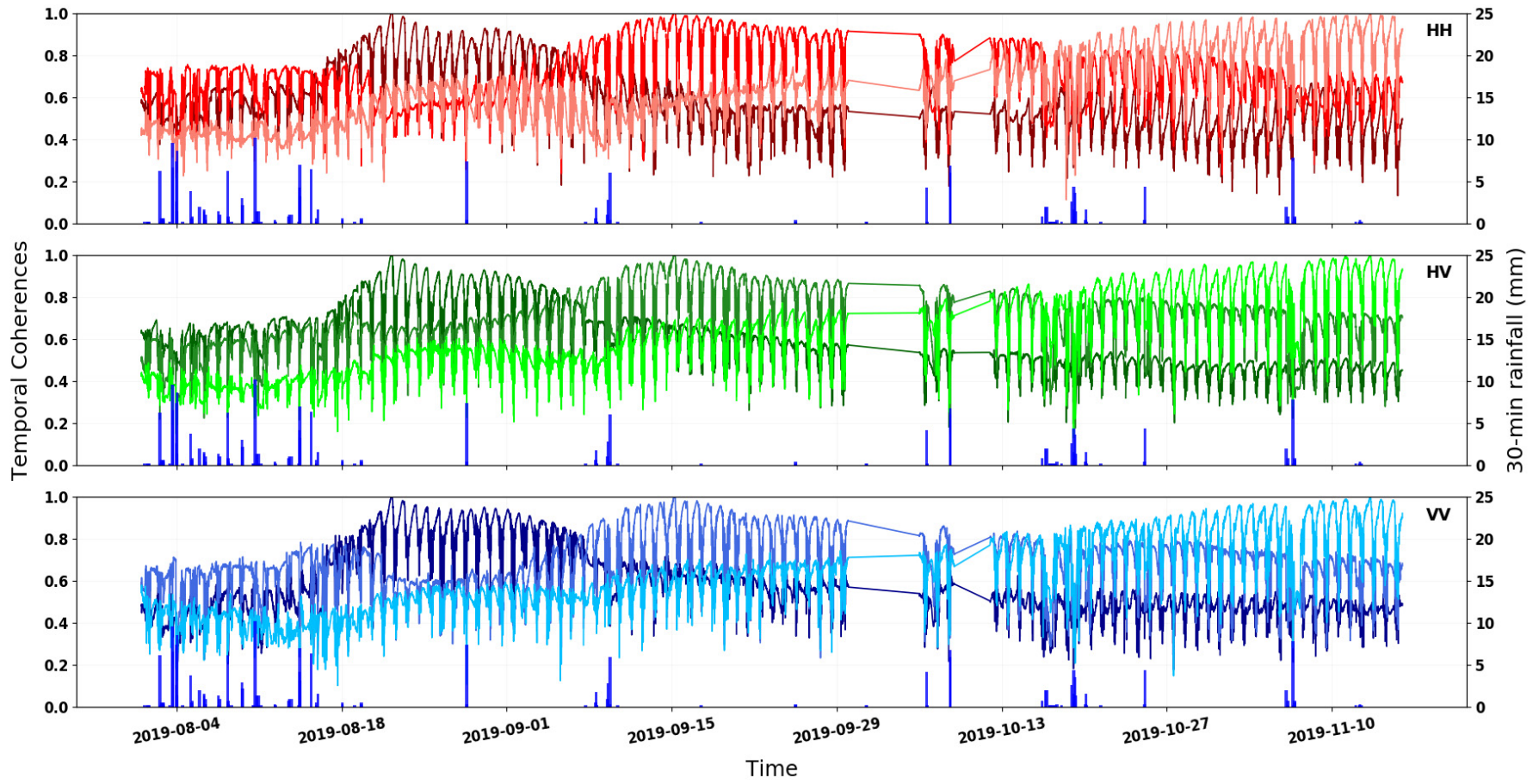

Fig. 8. Evolution of coherences computed over 2019 dry season with respect to three reference times: August 22, September, and November 13 at 6:00 A.M. represented, respectively, with light, intermediate, and dark colors for the three polarizations $\mathrm{HH}, \mathrm{HV}$, and VV in each line, overlaid with 30-min cumulative rainfall measurements. 

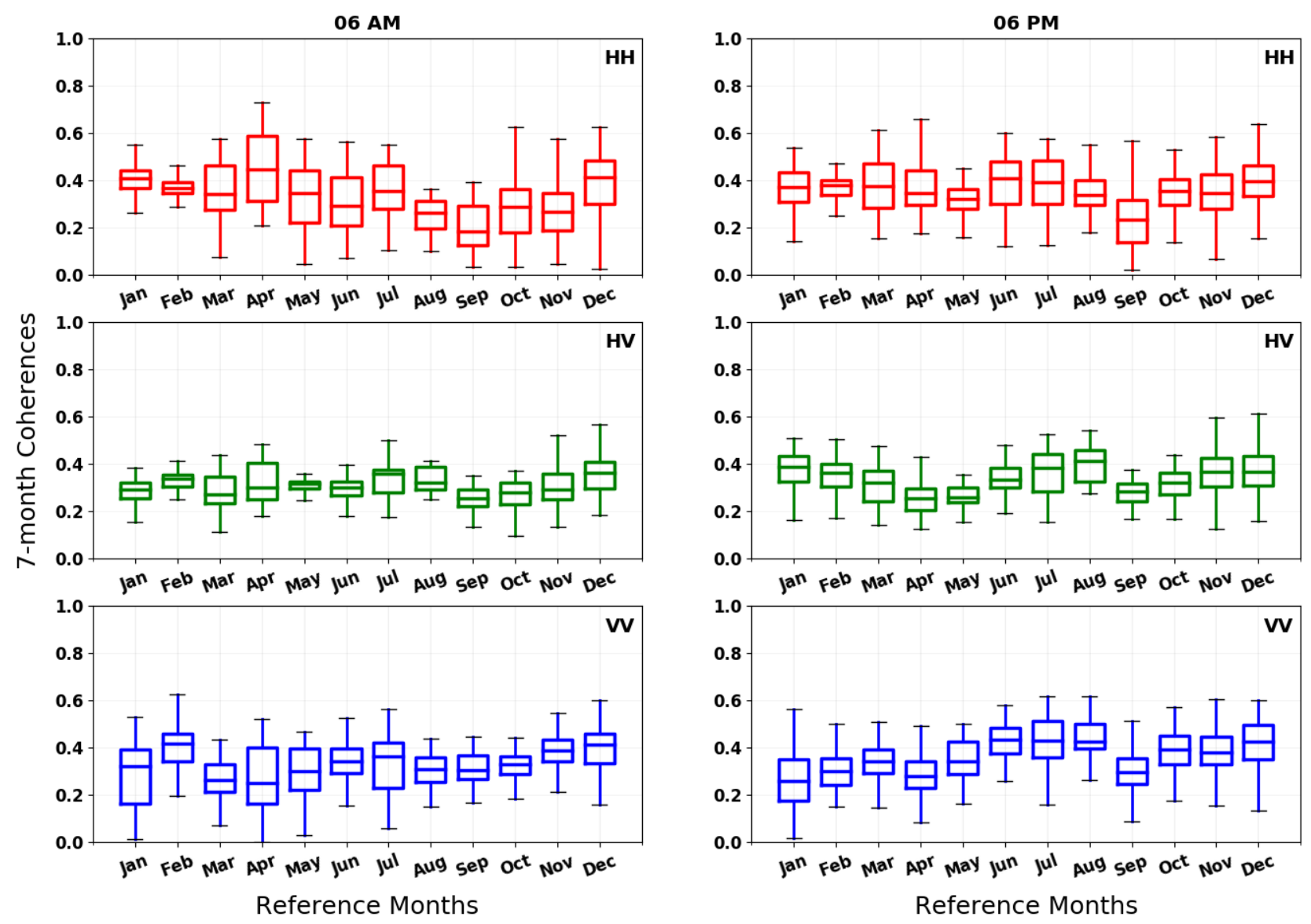

Fig. 9. Evolution of whisker boxes representing the statistical distribution of seven-month coherences as a function of the reference month over the year for (Left) 6:00 A.M. and (Right) 6:00 P.M. and the three polarizations HH, HV, and VV in each line.

with respect to each reference time. This approach provides the possibility to perform synchronous analysis with other processes such as the occurrence of rainy episodes, as shown in Fig. 8. It also highlights the typical diurnal cycles of the coherence variation exhibited by the oscillations observed in this same figure [7]. Although limited by possibly too specific effects due to the choice of a particular reference time, such representation enables to highlight the impacts of dated events on coherence evolution, as well as to explore the sources of the nonstationary trend observed during the dry period characterized by a dependence not only on temporal baselines but also on the selected year (see the differences raised above between 2018 and 2019 dry seasons). We have selected three different reference times in order to illustrate various trends in the coherence evolution. These references are purposely chosen to belong to nonrainy periods in order to better represent the dry season so that we can subsequently analyze the impact of rainfall occurrence on the coherence evolution, while the test time covers the whole season. As expected, these three curves are characterized by an overall decreasing trend, but with variable rates and modulations that can be mostly reported to rainy events. More specifically, four types of rain-related perturbing impacts can be distinguished: sudden perturbations when the loss of coherences is one-off, transient perturbations when a significant recovery time can be identified, irreversible perturbations when coherences remain low, and finally restorative perturbations when the on-going decreasing trend is interrupted by a gain of coherence. The latter case is rather unexpected and uncommon and can be observed, for instance, on the curve corresponding to the second reference time, when the test time varies toward the beginning of the season from around August 20. Although further analysis and in situ measurements would be required to investigate this phenomenon, the most likely explanation is that the occurrence of rainfall balances the vegetation and/or soil water status resulting from the drying-out or replenishment phases with respect to the reference time. The more pronounced increase in both copolarizations could indicate that this change in water status particularly affects the soil, the trunks, or both through double-bounce mechanisms since direct contributions from the ground or coupling interactions between ground and trunks are mostly negligible in the cross polarization. Besides, it is interesting to note that these perturbations can be related to the slope breaks observed in Fig. 7, especially to the one occurring around 25 days for the 2019 dry season. To conclude this section, we can highlight the importance of rainfall distribution during dry seasons since the corresponding coherences demonstrate a considerably higher and more complex sensitivity to these exceptional events, resulting in a less reproducible evolution over years. However, saturation 
TABLE IV

Correlation CoefFicients Between 12-Month CoHEREnCES CAlCulated AT 6:00 A.M. AND 6:00 P.M. AND Clas SIFIED BY THE SEASON

\begin{tabular}{|c|c|c|c|}
\hline & $\mathrm{HH}$ & $\mathrm{HV}$ & $\mathrm{VV}$ \\
\hline Dry Season & 0.448 & 0.214 & 0.125 \\
\hline Rainy Season & 0.357 & 0.360 & 0.287 \\
\hline
\end{tabular}

effects due to recurring heavy rainfall during rainy seasons induce comparable results from one year to another.

\section{7- AND 12-Month CoHEREnCES ANALYSiS}

\section{A. Seven-Month Coherences}

Considering the ability of BIOMASS to provide seven-month temporal decorrelation computed with a zero spatial baseline, new prospects for change detection based on these coherences can be foreseen, as long as the dynamic range between the undisturbed and disturbed states is sufficiently high with respect to their respective statistical dispersion. Leaving aside the very complex variety of possible disturbances (anthropogenic factors and meteorological or climatic events such as droughts), our study case allows the analysis of variable weather conditions over two consecutive years in the absence of any extraordinary events, offering the possibility to verify the prior requirement that seven-month coherences are indeed still significant in undisturbed conditions. The previous results presented in Section III support this assumption, although the seven-month coherence dispersion and especially values below the first quartile can be critical and would deserve a further characterization, as proposed in Sections VI-A1-VI-A2.

1) Monthly Representation Over the Year: Considering the importance of seasonality effects highlighted before, we can first analyze the yearly evolution of coherences displayed for each month depending on the reference time, although seven-month intervals do not match seasonal cycles. The resulting coherence distribution is shown in Fig. 9 for the three polarizations and both acquisition times. As expected, most values fall within the range of 0.2-0.4. Besides, the yearly evolution is rather a continuous one, whether for 6:00 A.M. or 6:00 P.M. with slightly higher values for the latter most of the time, although no correlation was observed between the two acquisition times. On the contrary, significant differences among polarizations are noticed and are more difficult to explain. Nevertheless, the cross-polarization channel appears to be the most appropriate for the change detection applications mentioned above. Indeed, the first quartile of the corresponding coherences is almost always higher than 0.2, which would enable a more accurate detection rate, still with the hypothesis of obtaining very low coherences in case of perturbations.

2) Classification Based on Cumulative Rainfall: To pursue the characterization of the seven-month coherences, the impact of rainfall can be directly investigated using a classification based on the amount of rainfall accumulated before both the reference and test times corresponding to each coherence
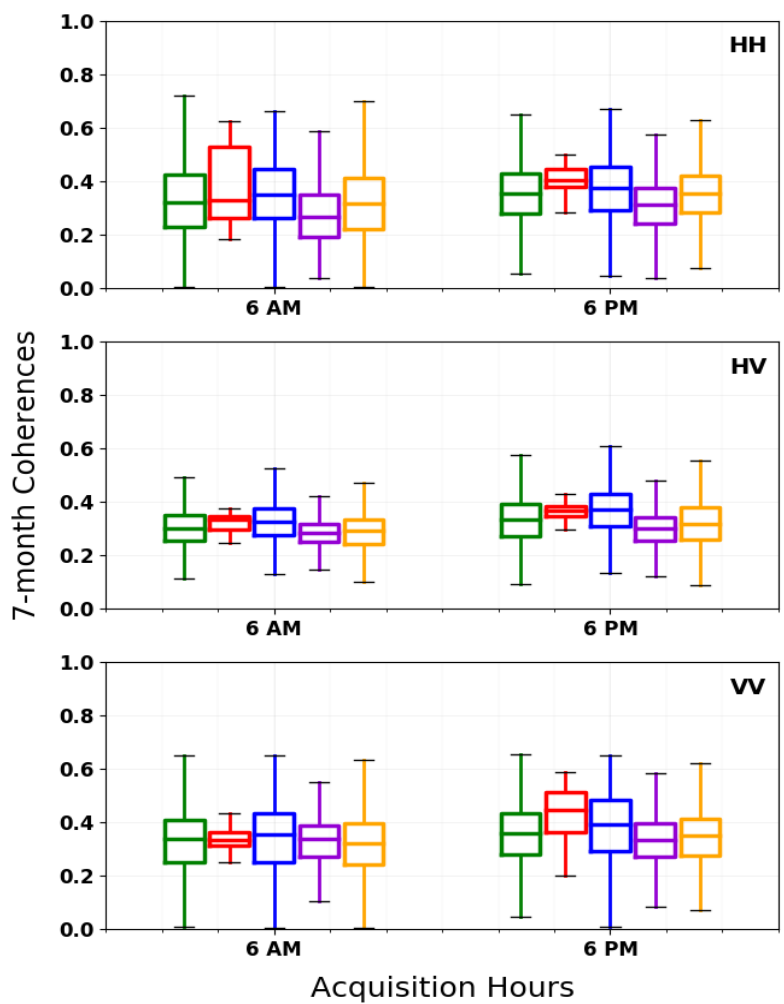

Fig. 10. Whisker boxes representing the statistical distribution of seven-month coherences (green) and their classification over the four defined subsets: dry-dry (red), rainy-rainy (blue), dry-rainy (purple), and remaining subset (orange) for both acquisition hours and the three polarizations $\mathrm{HH}, \mathrm{HV}$, and VV in each line.

value. To this end, we start by defining two designations. On one hand, an acquisition is considered to be part of a so-called dry period if the cumulative rainfall measured over its 15 previous days is less than $30 \mathrm{~mm}$ and that measured during the last two days is zero. On the other hand, an acquisition is considered to belong to a so-called rainy period if the cumulative rainfall measured over its 15 previous days is greater than $50 \mathrm{~mm}$ and that measured during the last two days is nonnull. Based on these two designations, we classify the coherence values into four subsets. If both reference and test times belong to the dry period, the corresponding coherence is assumed to be part of the first subset (dry-dry subset). When both reference and test times belong to the rainy period, the corresponding coherence is assigned to the second subset (rainy-rainy subset). However, when one of the two acquisition times belongs to the dry period and the other to the rainy one, the coherence is allocated to the third subset (dry-rainy subset). Finally, the rest of coherence values are gathered in the last subset (remaining subset). This classification of the seven-month coherences is shown in Fig. 10. From the outset, it can be noticed that the dry-dry subset usually seems to have the narrowest spread, whereas the rainy-rainy subset and the rainy-dry one (involving two possible different conditions and physiological status) cannot be distinguished. This representation also confirms the slightly higher values at 6:00 P.M., whatever the polarization. Interestingly, it can also be highlighted that median values of the dry-dry coherences are the highest at 6:00 P.M., which would be the most 

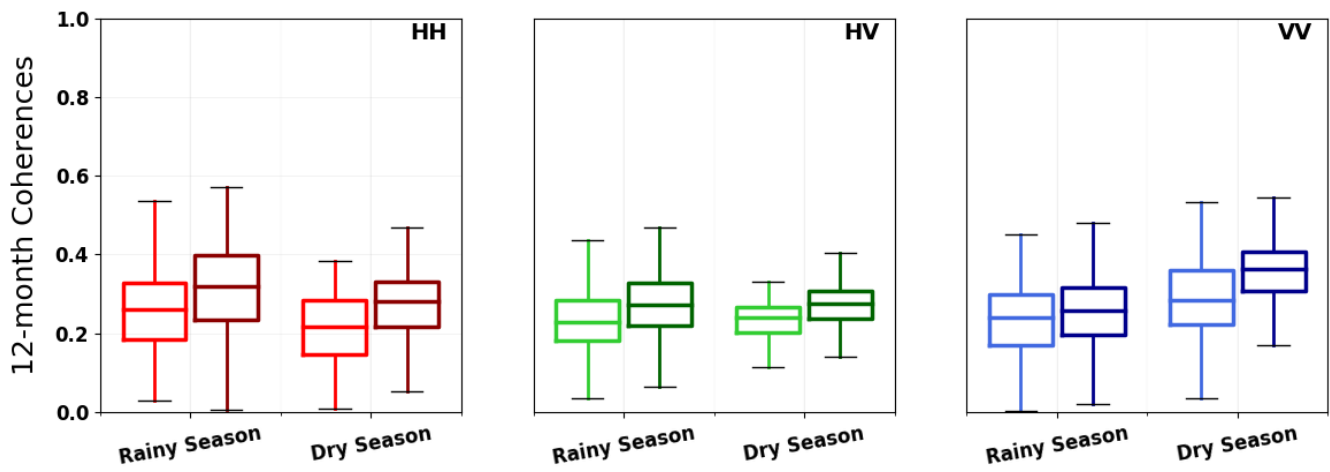

Fig. 11. Whisker boxes representing the statistical distribution of 12-month coherences classified as a function of seasons for (Left) 6:00 A.M. (light colored) and (Right) 6:00 P.M. (dark colored) and the three polarizations HH, HV, and VV in each column.
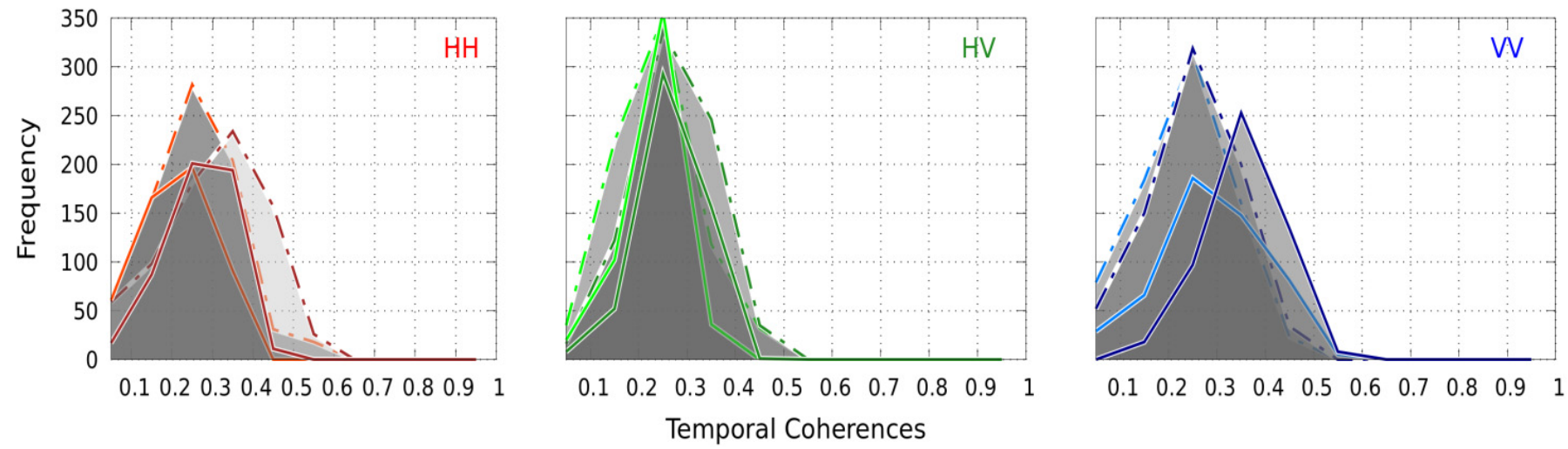

Fig. 12. Distributions of 12-month coherences classified by season for the three polarizations HH, HV, and VV. For each subfigure, the rainy and dry seasons are represented with dotted and plain lines and the 6:00 A.M. and 6:00 P.M. by light or dark colors.

appropriate to detect anomalies related, for instance, to exceptional drought periods, assuming again that such disturbances will induce very low coherences compared to a typical reference state.

\section{B. 12-Month Coherences}

To deepen our understanding of seven-month coherences, 12-month intervals can be considered to exploit annual cycles related to physical parameters. As performed previously, several representations and classifications are used in the following to better isolate the effects of precipitations.

1) Classification Based on Seasons: We first represent the 12-month coherences depending on the seasonality of the reference month, as shown in Fig. 11. As detailed in Section II-A, the global rainy season is considered to be constituted of the months of January, February, April, May, July, November, and December, whereas March, August, September, and October represent the global dry season. Coherence values corresponding to the global rainy season are characterized by a greater dispersion except for VV where the dispersion is almost the same for both seasons. Medians of $\mathrm{HH}$ coherences are slightly higher in the rainy season than in the dry one. This could be explained by the fact that the forest soil is saturated during the rainy season and therefore remains relatively coherent, unlike during the dry season where low amount of rainfall can cause a significant drop in coherence values, as highlighted in Section IV. We note once again that coherences computed at 6:00 P.M. are slightly higher than those at 6:00 A.M. Fig. 12 shows the distributions corresponding to the whisker boxes of Fig. 11 and confirms that the 12-month coherences computed at 6:00 P.M. are generally higher than those at 6:00 A.M. whatever the season and polarization. These distributions also highlight that the dispersions obtained for $\mathrm{HH}$ and $\mathrm{HV}$ are significantly higher in the rainy season, while this is not the case for VV. Interestingly, the significant correlation between the 12-month coherences computed at 6:00 A.M. and 6:00 A.M. (see Table IV) is worth noticing. On the contrary to the seven-month coherences case (see Section VI-A1), these significant values at 12 months reflect the matching between the days for which coherences at 6:00 A.M. or 6:00 P.M. are found above or below the mean, letting us hypothesize the manifestation of annual cycles at the origin of more similar conditions between days separated by 12 than by seven months.

2) Monthly Representation Over the Year: A further way for analyzing the 12-month coherences is by displaying them depending on their reference month as shown in Fig. 13, into which the monthly cumulative rainfall for both the reference and test month is overlaid. Overall, medians of the 12-month coherences range between 0.2 and 0.4. More precisely, coherences computed during the rainiest months (April, May, and December) are characterized by wider whisker boxes contrary to those computed on the driest months (March, September, and October) which are the narrowest. Curiously, coherences computed over March (small dry season in the middle of two rainy ones) represent the minimum over the whole year for the 

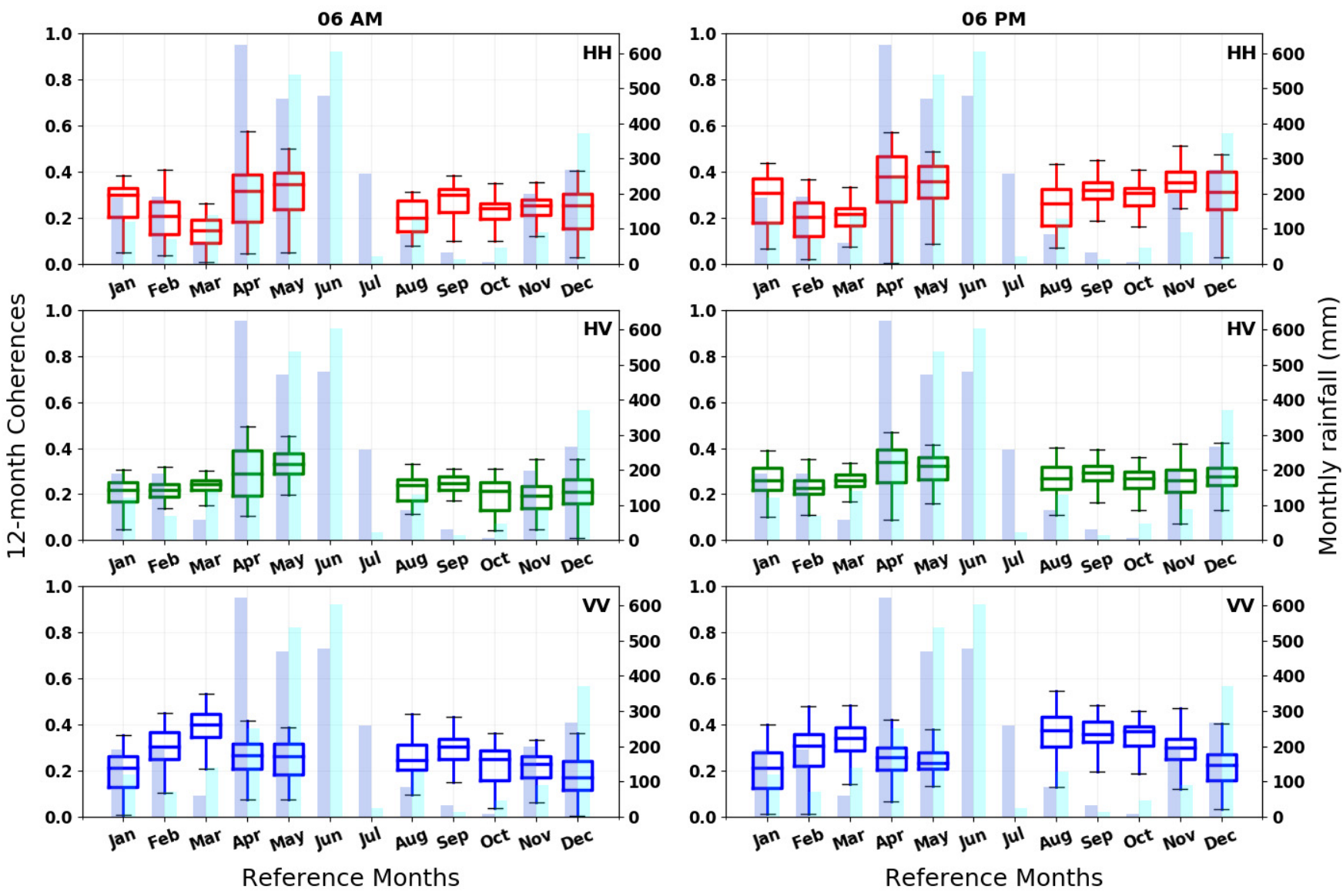

Fig. 13. Whisker boxes representing the statistical distribution of 12-month coherences as a function of the reference month over the year, overlaid with the monthly cumulative rainfall for both the reference (dark blue) and test (light blue) months, for (Left) 6:00 A.M. and (Right) 6:00 P.M. and the three polarizations $\mathrm{HH}, \mathrm{HV}$, and VV in each line.

$\mathrm{HH}$ polarization and the maximum for the VV polarization for both acquisition times. Finally, coherences over the two rainiest months (April and May) are the highest in $\mathrm{HH}$ and the cross polarization.

3) Classification Based on Cumulative Rainfall: To better illustrate the effects of rain on the classification of the 12-month coherences, we have classified their values depending on the cumulative rainfall recorded before both reference and test times as performed for the seven-month coherences in Section VI-A2. This classification is shown in Fig. 14. It can be immediately noticed that whisker boxes of coherences corresponding to the dry-dry subset are always the narrowest, while those corresponding to the rainy-rainy subset are generally the widest. Moreover, coherence medians of all subsets are almost aligned for each hour except for the median of coherences belonging to the dry-dry period in VV at 6:00 P.M. which is the highest. Again, medians of coherences computed at 6:00 P.M. are slightly higher than those at 6:00 A.M. This observation was noted several times throughout the study and only with regard to very long-term coherences. It is important to note that a similar number of looks have been found for both acquisition times, which confirms a physical explanation behind these observed differences. When analyzing the evolution of wind measurements recorded around both 6:00 A.M. and 6:00 P.M. throughout the year in Fig. 4, we note from the outset that wind is always stronger at 6:00 P.M. than at 6:00 A.M. This supports the idea that, on the one hand, short-term coherences are more sensitive to quasi-random disturbances related to wind variations, and on the other hand, long-term coherences are rather dominated by other physical phenomena related to vegetation growth and cumulative variations in water conditions [25].

\section{DISCUSSION}

To better put into perspective the scope of the presented results and especially their applicability in the frame of future spaceborne missions including BIOMASS, three important points can be discussed. First, the possible impact of rain on the antennas is a common issue of ground- and tower-based systems, which obviously cannot be transposed to the spaceborne ones. Previous tests during the experiment setup [7] have shown that simultaneous measurements with heavy rainfall events could severely impact the antennas, but their recovery is very short (less than few seconds in most cases, depending on dry-up conditions). However, this issue needs to be checked whenever possible in our analysis. Indeed, the filtering of rainy events for the present results (if the 15-min acquisition cycle has coincided with a rainfall event) leads to a lower dispersion of the coherence values but with slightly higher median values. To preserve the number of samples (especially for the longest baselines limited by the available pairs of dates), we preferred 

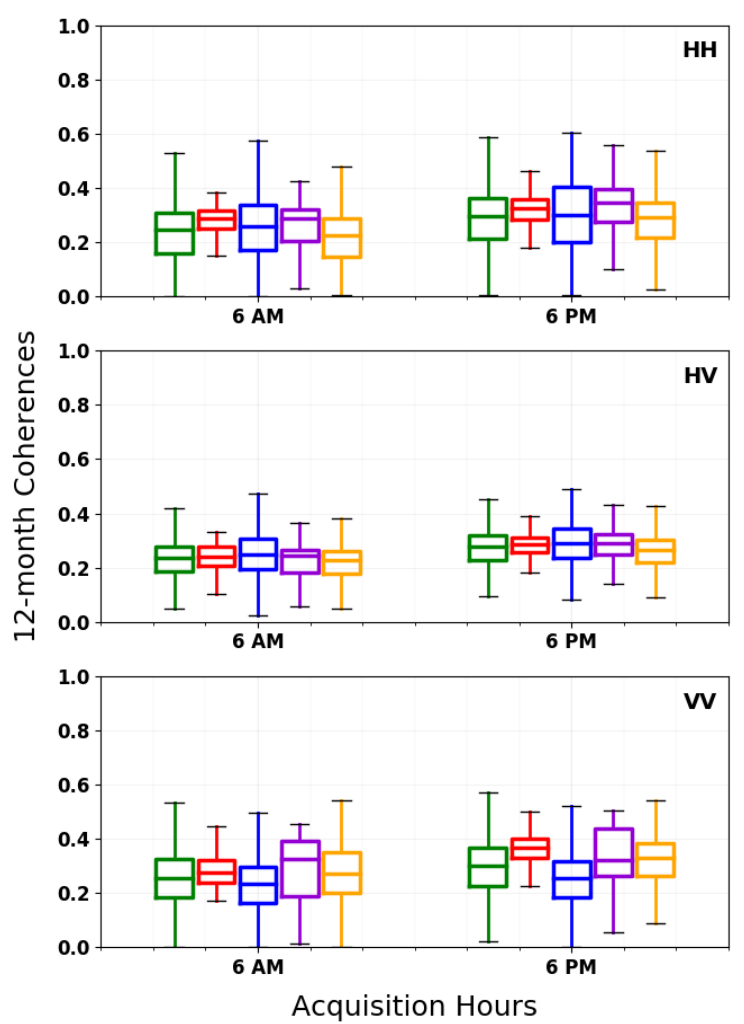

Fig. 14. Whisker boxes representing the statistical distribution of 12-month coherences (green) and their classification over the four defined subsets: dry-dry (red), rainy-rainy (blue), dry-rainy (purple), and remaining subset (orange), depending on the acquisition hour and for the three polarizations $\mathrm{HH}, \mathrm{HV}$, and VV in each line.

to retain all acquisitions, providing thereby a worst case scenario with a slight underestimation of coherences and a more significant overestimation of dispersion. For the applicability to spaceborne configurations, we recommend to give more importance to statistical values close to the median than to the full spread of coherence values.

Second, it is also worth to recall that a remaining system decorrelation may also underestimate our evaluation of true temporal coherences. Indeed, although the compensation presented in Section II-B was performed to minimize these effects, the temporal coherence computed for pixels corresponding to the tower structure varies around 0.9 for 14 -month intervals. Nonetheless, it remains difficult to attribute this error to the system decorrelation or to possible physical changes on the tower surface (i.e., superficial humidity or dust), and the most important is that these uncertainties would not provide a bias larger $10 \%$.

The last point concerns the tower height limitation of this experiment (about $55 \mathrm{~m}$ ), resulting in a range of incidence angles throughout the swath much wider than for a spaceborne configuration. Considering also the forest edge discontinuity due to the presence of the tower, we have chosen a more representative region between 45 and $60 \mathrm{~m}$ from the tower base, hence a range of incidence angles with respect to a virtual horizontal ground of about $\left[40^{\circ}, 48^{\circ}\right]$ instead of the $\left[25^{\circ}, 32^{\circ}\right]$ planned for the BIOMASS mission. In terms of impacts on coherence estimates, the question is not straightforward but can be addressed through the dependence of the main scattering mechanisms to the incidence angle [26], [27], given that these mechanisms (e.g., volume or double-bounce interaction with the ground) involve different components of the vegetation not equally sensitive to temporal decorrelation. Nonetheless, the use of polarization to select or combine scattering mechanisms can be emphasized here, and the rather similar results and trends we show among the three polarizations provide a relevant indication that in our case (i.e., tropical dense forest), the dependence of coherence evolution to incidence angle would be limited.

\section{CONCLUSION}

The presented work focuses on the investigation of P-band temporal decorrelation based on unprecedented polarimetric time series extending over 617 days. The study enables not only to analyze the coherence evolution over a range of temporal baselines reaching 14 months but also to further explore the coherence variability with respect to specific intervals of interest. Indeed, the study highlights the variable distribution of the 18-day coherences throughout the year, which can be considered as an indicator of a likely variability for lower temporal baselines (three and six days for BIOMASS). Such variability indicates that unequal performances of BIOMASS tomographic products could be expected over the world and thus emphasizes the importance of ascending and descending products to mitigate resulting decorrelation errors. Moreover, the study confirms and consolidates previous results about the differences raised between decorrelation patterns over dry and rainy periods up to three months and further underlines the high sensitivity of coherences derived during the dry season with respect to rainfall. Indeed, the cross analysis with rainfall distribution indicates that the occurrence of a rainy event during the dry season has a stronger impact on the coherence evolution than during the rainy season, with either a punctual or a definitive loss of coherence, or less commonly a raise in coherence values. In addition, a stronger sensitivity of coherences during dry seasons has also been confirmed with respect to longer temporal baselines available in the present study. A possible hypothesis behind such sensitivity lies on progressive inner changes driven by the vegetation drying out, although this hypothesis would require additional in situ measurements specifically designed for vegetation water content and dendrometric variations.

For temporal baselines beyond three months, differences between dry and rainy seasons are lower, but we can still observe seasonality effects on the seven-month coherences with median values ranging from 0.2 to 0.4 . Similarly, medians of the 12-month coherences are higher than 0.2 but do not reveal any evidence of annual cycles, although the correlations between these coherences at 6:00 A.M. and 6:00 P.M. may enable to highlight annual variations. In addition, values calculated at 6:00 P.M. are slightly higher than those at 6:00 A.M., which could be imputed to a more stable water status in the evening and greater robustness of the long-term coherences with respect to wind effects. As for the seven-month coherences, the dispersion around median values can be partially explained by the cumulative rainfall amount, yet the annual coherences stand out by $25 \%$ of 
computed values falling below 0.2 with minimums reaching zero. These differences are particularly interesting since they demonstrate that seven-month coherences do not saturate and are high enough to detect anomalies regarding the forest status, whether impacted by environmental conditions (such as droughts) or sudden perturbations due to storms or human activities. It should be recalled that BIOMASS will measure temporal coherences with a zero spatial baseline only between global cycles reached every seven months. As demonstrated in the present work, these coherences could be truly relevant to consolidate standard approaches of change detection based on variations in backscattering coefficients. However, BIOMASS interferometric coherences will convey aggregated information of both temporal and geometric decorrelations, the latter being mainly conditioned by the vegetation height.

In addition, this article highlights the relationship between temporal coherences and intensity correlations. Consequently, the current prediction of the latter could not only be used as a performance indicator of the multitemporal filtering of backscattering coefficients but also as a proxy of temporal coherences in order to exploit their sensitivity to forest changes across BIOMASS time scales. Considering the time remaining before BIOMASS product delivery, additional work will be devoted to consolidate our understanding of the valuable intrinsic and mutual information generated from radar measurements, including temporal and spatial coherences, intensity correlations, and backscattering coefficients. Beyond the applications related to space missions, the pursuit of these analyses will contribute to improving our understanding of the underlying physics, in particular through the consolidation of in situ measurements dedicated to the characterization of tree growth and water status conditions.

\section{ACKNOWLEDGMENT}

The authors would like to thank the French National Space Agency (CNES) for supporting activities in the framework of the TOSCA Research Program. They would like to thank the European Space Agency (ESA), the European Space Research and Technology Centre (ESTEC), and the European Space Research Institute (ESRIN) teams for following our research activity as well as people at Eco Fog for their contribution to the success of the Paracou research station. They would also like to thank the reviewers for their relevant comments and overall interest.

\section{REFERENCES}

[1] T. Le Toan et al., "The BIOMASS mission: Mapping global forest biomass to better understand the terrestrial carbon cycle," Remote Sens. Environ., vol. 115, no. 11, pp. 2850-2860, Nov. 2011.

[2] S. Quegan et al., "Report for mission selection: Biomass. Science," Eur. Space Agency, Noordwijk, The Netherlands, Tech. Rep. ESA SP 1324/1, 2012, p. 193, vol. 3 .

[3] S. Quegan et al., "The European space agency BIOMASS mission: Measuring forest above-ground biomass from space," Remote Sens. Environ., vol. 227, pp. 44-60, Jun. 2019.

[4] T. Le Toan, S. Quegan, I. Woodward, M. Lomas, N. Delbart, and G. Picard, "Relating radar remote sensing of biomass to modelling of forest carbon budgets," Climatic Change, vol. 67, nos. 2-3, pp. 379-402, Dec. 2004.

[5] L. Villard, T. Le Toan, D. H. T. Minh, S. Mermoz, and A. Bouvet, "Forest biomass from radar remote sensing," in Land Surface Remote Sensing in Agriculture and Forest. Amsterdam, The Netherlands: Elsevier, 2016, pp. 363-425.
[6] Guyaflux Description. Accessed: Nov. 10, 2020. [Online]. Available: https://paracou.cirad.fr/website

[7] C. Albinet et al., "TropiSCAT: A ground based polarimetric scatterometer experiment in tropical forests," IEEE J. Sel. Topics Appl. Earth Observ. Remote Sens., vol. 5, no. 3, pp. 1060-1066, Jun. 2012.

[8] H. T. M. Dinh et al., "Ground-based array for tomographic imaging of the tropical forest in P-band," IEEE Trans. Geosci. Remote Sens., vol. 51, no. 8, pp. 4460-4472, Aug. 2013.

[9] A. Hamadi, L. Villard, P. Borderies, C. Albinet, T. Koleck, and T. Le Toan, "Comparative analysis of temporal decorrelation at P-band and low L-band frequencies using a tower-based scatterometer over a tropical forest," IEEE Geosci. Remote Sens. Lett., vol. 14, no. 11, pp. 1918-1922, Nov. 2017.

[10] Y. Bai, S. Tebaldini, D. H. T. Minh, and W. Yang, "An empirical study on the impact of changing weather conditions on repeat-pass SAR tomography," IEEE J. Sel. Topics Appl. Earth Observ. Remote Sens., vol. 11 , no. 10 , pp. 3505-3511, Oct. 2018.

[11] A. Lopes and F. Sery, "Optimal speckle reduction for the product model in multilook polarimetric SAR imagery and the wishart distribution," IEEE Trans. Geosci. Remote Sens., vol. 35, no. 3, pp. 632-647, May 1997.

[12] S. Quegan and J. J. Yu, "Filtering of multichannel SAR images," IEEE Trans. Geosci. Remote Sens., vol. 39, no. 11, pp. 2373-2379, Nov. 2001.

[13] A. Hamadi et al., "Temporal coherence of tropical forests at P-band: Dry and rainy seasons," IEEE Geosci. Remote Sens. Lett., vol. 12, no. 3, pp. 557-561, Mar. 2015.

[14] C. Albinet et al., "First results of AfriScat, a tower-based radar experiment in African forest," in Proc. IEEE Int. Geosci. Remote Sens. Symp. (IGARSS), Jul. 2015, pp. 5356-5358.

[15] AfriScat Final Report, document ESA Contract $N^{\circ} 4000111758 / 14 / \mathrm{NL}$ /FF/GP, 2018.

[16] S. El Idrissi Essebtey et al., "Temporal decorrelation of tropical dense forest at C-band: First insights from the TropiScat-2 experiment," IEEE Geosci. Remote Sens. Lett., vol. 17, no. 6, pp. 928-932, Jun. 2020.

[17] S. E. I. Essebtey, L. Villard, P. Borderies, T. Koleck, B. Burban, and T. L. Toan, "Comparative study of temporal decorrelation at P, L and C-bands: First insights from the tropiscat-2 experiment," in Proc. Medit. Middle-East Geosci. Remote Sens. Symp. (MGARSS), Mar. 2020, pp. $246-249$.

[18] A. Hamadi et al., "Temporal survey of polarimetric P-band scattering of tropical forests," IEEE Trans. Geosci. Remote Sens., vol. 52, no. 8 , pp. 4539-4547, Aug. 2014.

[19] J. W. Goodman, "Some fundamental properties of speckle," J. Opt. Soc. Amer, vol. 66, no. 11, pp. 1145-1150, 1976.

[20] C. Gelas, L. Villard, L. Ferro-Famil, L. Polidori, T. Koleck, and S. Daniel, "Multi-temporal speckle filtering of polarimetric P-band SAR data over dense tropical forests: Study case in French guiana for the BIOMASS mission," Remote Sens., vol. 13, no. 1, p. 142, Jan. 2021.

[21] R. Touzi, A. Lopes, J. Bruniquel, and P. W. Vachon, "Coherence estimation for SAR imagery," IEEE Trans. Geosci. Remote Sens., vol. 37, no. 1, pp. 135-149, Jan. 1999.

[22] J. Bruniquel and A. Lopes, "Multi-variate optimal speckle reduction in SAR imagery," Int. J. Remote Sens., vol. 18, no. 3, pp. 603-627, Feb. 1997.

[23] J. S. Lee, L. Jurkevich, P. Dewaele, P. Wambacq, and A. Oosterlinck, "Speckle filtering of synthetic aperture radar images: A review," Remote Sens. Rev., vol. 8, no. 4, pp. 313-340, Feb. 1994.

[24] S. Foucher and C. Lopez-Martinez, "Analysis, evaluation, and comparison of polarimetric SAR speckle filtering techniques," IEEE Trans. Image Process., vol. 23, no. 4, pp. 1751-1764, Apr. 2014.

[25] C. Stahl, B. Burban, F. Bompy, Z. B. Jolin, J. Sermage, and D. Bonal, "Seasonal variation in atmospheric relative humidity contributes to explaining seasonal variation in trunk circumference of tropical rainforest trees in French guiana," J. Tropical Ecol., vol. 26, no. 4, pp. 393-405, Jul. 2010.

[26] M. M. d'Alessandro and S. Tebaldini, "Phenomenology of P-band scattering from a tropical forest through three-dimensional SAR tomography," IEEE Geosci. Remote Sens. Lett., vol. 9, no. 3, pp. 442-446, May 2012.

[27] L. Villard, P. Borderies, T. Le Toan, T. Koleck, and C. Albinet, "Topography effects on forest radar scattering, consequences on biomass retrieval," in Proc. IEEE Int. Geosci. Remote Sens. Symp. (IGARSS), Jul. 2010, pp. 60-63. 